Revista Española de Antropología Americana ISSN: 0556-6533

https://doi.org/10.5209/REAA.61979

\title{
El desarrollo de la sociedad urbana en el Altiplano Central mexicano durante el Formativo y la transición al Clásico
}

\author{
David M. Carballo ${ }^{1}$
}

Recibido: 10 de marzo de 2017 / Aceptado: 3 de julio de 2017

Resumen. Durante los dos milenios denominados periodos Formativo (1500 a.C.-100 d.C.) y Clásico (100-650 d.C.), las sociedades del Altiplano Central mexicano realizaron muchos de los cambios claves típicamente asociados con la complejidad social pre-moderna. Estos incluyen la transición a una vida agrícola y sedentaria, la creación de centros urbanos con arquitectura monumental, diversificación de la producción, intensificación de redes de intercambio, diferenciación de estatus y desigualdad social; así como la apariencia de dioses, símbolos y espacios sagrados de un sistema religioso compartido y de larga duración. Este resumen se enfoca en tales transiciones, sobre todo durante el intervalo aproximado comprendido entre el 600 a.C. y 250 d.C., que cubre los períodos designados como finales del Formativo Medio, Formativo Tardío, Formativo Terminal y el inicio del Clásico Temprano.

Palabras clave: sedentarismo, urbanización, religión, precolombino, México.

\section{[en] The Development of Urban Society in the Central Highlands of Mexico during the Formative Period and Transition to the Classic Period}

\begin{abstract}
During the two millennia designated the Formative period (1500 BC - AD 100) and the Classic period (AD 100-650), societies of the central Mexican highlands undertook many of the key transformations typically associated with premodern social complexity. These include the transition to agricultural and sedentary lifeways; the creation of urban centers with monumental architecture; the diversification of economic production, intensification of exchange networks, differentiation of status, and heightened social inequality; and the formalization of depictions of gods, symbols, and sacred spaces of a religious system that was widely shared and long lasting. This summary of the period focuses on such transitions, especially during the interval of ca. $600 \mathrm{BC}-\mathrm{AD} 250$, covering the periods designated in central Mexico as the end of the Middle Formative, the Late Formative, the Terminal Formative, and the beginning of the Early Classic.
\end{abstract}

Keywords: sedentary lifestyle, urbanization, religion, Precolumbian, Mexico.

Sumario. 1. Introducción. 2. Líneas de investigación arqueológicas y patrones regionales. 3. El sedentarismo y el urbanismo inicial del Formativo Temprano y Medio. 4. Ciudades y dioses en el Formativo Tardío y Terminal. 5. Las transiciones al período Clásico. 6. Referencias.

\footnotetext{
1 Departamento de Arqueología, Universidad de Boston. carballo@bu.edu
} 


\section{Introducción}

Hoy en día el Altiplano Central mexicano es bien conocido por su cercanía con la zona metropolitana de la Ciudad de México, siendo una de las más pobladas del mundo. Los procesos de urbanización tienen una larga trayectoria en la región, iniciándose aproximadamente a mitad del primer milenio a.C., durante el Formativo Medio (900-500 a.C.) y el Formativo Tardío (500-100 a.C.), y continuando durante el periodo Clásico (150-650 d.C.) con el caso más conocido al nivel mundial, la ciudad de Teotihuacan, la cual fue la más poblada de las Américas durante su apogeo. En el presente trabajo, se resume este periodo de transformación en el centro de México con un enfoque especial en temas del desarrollo de la sociedad urbana. Se incorporan en el presente trabajo otros tratamientos sintetizados más recientes de este periodo y se proporcionan detalles acerca de otros temas clave (Carballo 2016; Cowgill 2015; García Cook 2014; Plunket y Uruñuela 2005, 2012).

Por su composición demográfica y fisiográfica, el centro de México cuenta con una amplia diversidad de grupos étnicos y regiones ambientales. En el caso del primero, las autoras Patricia Plunket y Gabriela Uruñuela (2012) lo han caracterizado como una «costura cultural» que vio la fusión de tradiciones del este y del oeste de Mesoamérica durante el periodo Formativo. En el caso del segundo, se cuenta con una meseta o altiplano como su corazón fisiográfico y unas regiones más bajas en áreas adyacentes (Figura 1). En términos locales, el Altiplano Central se caracteriza como «tierra fría», con elevaciones típicamente superiores a los $2000 \mathrm{~m}$, mientras que otras áreas más bajas (entre aproximadamente 1000-2000 m), se clasifican como tierra templada. La precipitación anual es variable, pero generalmente llueve más por el sur del altiplano, alcanzando más de $1000 \mathrm{~mm}$ y está concentrada durante la

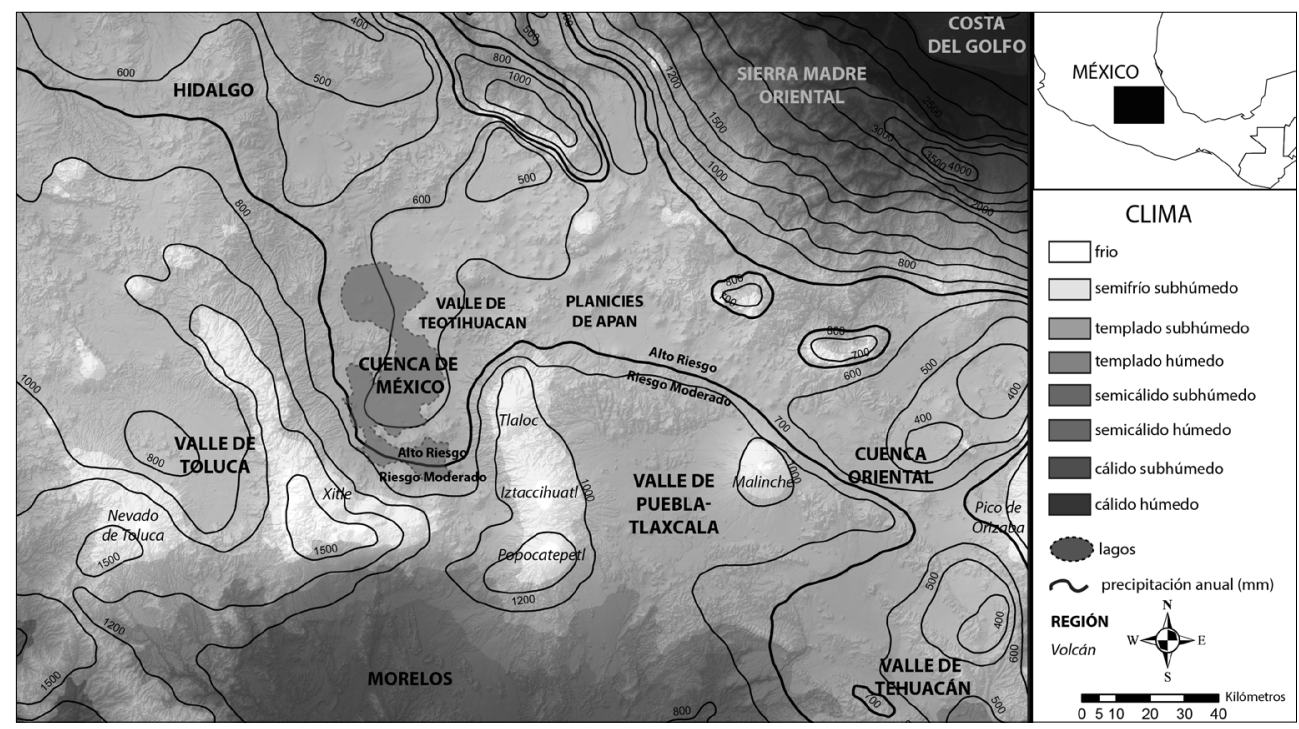

Figura 1. Mapa climatológico del centro de México mostrando zonas climáticas, precipitación anual y algunas regiones y volcanes. Datos obtenidos del Instituto Nacional de Estadística y Geografía (INEGI), México. 
temporada de lluvias entre los meses de mayo y octubre. En el principal estudio realizado con un enfoque cultural-ecologista, Sanders et al. (1979: 82-84) clasificaron las áreas con $700 \mathrm{~mm}$ de precipitación anual como zonas de riesgo para la agricultura basada en el maíz. Sin embargo, en los centros urbanos, incluyendo Teotihuacan que fueron fundados en áreas con menos lluvia de la postulada de $700 \mathrm{~mm}$, baja esta cifra, con la presencia de manantiales, humedales, lagunas y otros tipos de agua permanente, siendo clave en tales casos. Adicionalmente, es necesario considerar el hecho de que el paisaje actual no es el mismo que el del pasado (Sánchez Pérez et al. 2013) y los modelos cultural-ecologistas tienen que tomar en cuenta el impacto de cambios climáticos y transformaciones antropogénicas.

En cuanto a la diversidad poblacional, el centro de México fue ocupado por diferentes grupos étnicos durante el periodo prehispánico, con descendientes actuales entre las comunidades indígenas y mestizas. En el siglo XVI, los idiomas predominantes del Altiplano fueron el náhuatl (lengua franca del imperio azteca) y el otomí. Este segundo es parte de la familia otomangue, junto con el mixteco y el zapoteco, concentrados al sur de las tierras altas de México, y con una mayor antigüedad en la región. El tema de la introducción del náhuatl sigue siendo tema de debate pero, para el estudio de tiempos anteriores, los especialistas generalmente consideran las concordancias tanto como las divergencias entre los grupos mejor conocidos de fases históricas y el registro arqueológico.

\section{Líneas de investigación arqueológicas y patrones regionales}

Las líneas de evidencia disponibles para reconstruir las transformaciones sociales entre el Formativo y el Clásico son múltiples. Aunque nos faltan textos primarios, excepto por los muy pictográficos glifos teotihuacanos que a pesar de que cuentan con más de un siglo de investigaciones arqueológicas e iconográficas aún no han sido traducidos, la cobertura del territorio por parte de recorridos arqueológicos regionales es buena y nos permite tener reconstrucciones demográficas bien constatadas. Los proyectos de excavación continúan mejorando nuestra comprensión de las dinámicas comunitarias dentro de las ciudades, pueblos y aldeas, así como los distintos niveles del urbanismo. Los sitios individuales revisados en este trabajo se han priorizado por la calidad de la información disponible y también para poder presentar una mezcla de tamaños de comunidades con el fin de contemplar un escenario compuesto de centros urbanos y zonas más rurales (Figura 2).

En el centro de México han sido realizados una serie de proyectos regionales, siendo el más ambicioso el llevado a cabo en la Cuenca de México por parte de Sanders, Parsons y colegas. Estos trabajos comenzaron como parte del Proyecto Valle de Teotihuacan en 1960 y continuaron en otras partes de la cuenca a través de la década de 1970, culminando en una publicación sintética (Sanders et al. 1979) y otras monografías que han ido saliendo hasta los años 2000 (Gorenflo y Sanders 2007). En conjunto se han registrado, de forma sistemática, más de $4000 \mathrm{~km}^{2}$ de la Cuenca de México. Grandes porciones del área se encuentran actualmente debajo de la zona metropolitana de la Ciudad de México y en algunos casos, gracias a los esfuerzos de dichos proyectos, estos datos son los pocos que tenemos accesibles. De las ocupaciones formativas documentadas por los recorridos en la cuenca, varios fueron excavados por Tolstoy, proporcionando un refinamiento de la cronología regional 


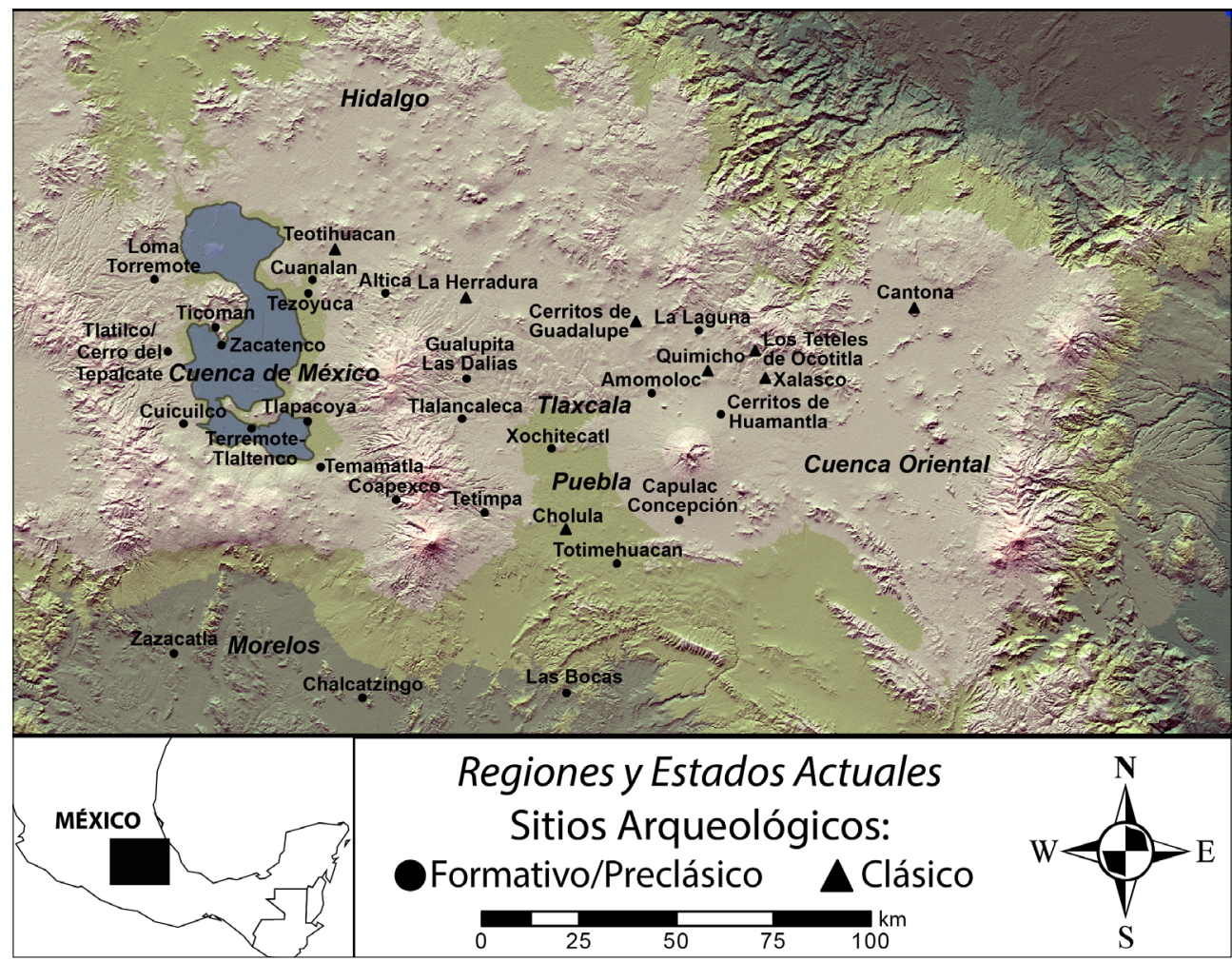

Figura 2. Centro de México con algunos sitios arqueológicos de los periodos Formativo y Clásico.

(Tolstoy et al. 1977). Entre los proyectos centrados en los centros urbanos de mayor importancia se incluyen las décadas de investigación en Cuicuilco, asentamiento que dominó el sur de la cuenca durante gran parte del Formativo (Pastrana y Ramírez 2012), y en Teotihuacan, el cual controló el norte de la cuenca siendo el centro más poderoso en Mesoamérica durante el Clásico (Cowgill 2015).

En la región de Puebla-Tlaxcala, un proyecto multidisciplinario que supuso la colaboración entre arqueólogos mexicanos e investigadores alemanes de la Fundación Alemana para la Investigación Científica (FAIC), produjo un gran corpus de datos y publicaciones arqueológicas, climatológicas, etnográficas, geológicas e históricas en los años 60s y 70s. Muchos de estos proyectos fueron muy relevantes, destacando el Proyecto Arqueológico Puebla-Tlaxcala (PAPT), que se inició en 1972 bajo la dirección de García Cook (1976). El recorrido del proyecto cubrió $4000 \mathrm{~km}^{2}$ de Puebla-Tlaxcala, y el Proyecto Arqueológico del Norte de Tlaxcala (PANT) añadió otros $2000 \mathrm{~km}^{2}$ a este total, que fue publicado por Merino Carrión (1989). Entre los centros urbanos de mayor importancia en la región durante el Formativo se incluye a Xochitecatl (Serra Puche 2012) y Tlalancaleca (García Cook 1973), ambos en el sur, y durante el Clásico están presentes Cholula (Plunket y Uruñuela 2005) y Cantoná (García Cook 2003) al sur y al norte, respectivamente, del actual estado de Puebla.

Otros estudios regionales de menor escala también merecen mención por su valor para comprender los patrones demográficos del período Formativo y Clásico en 
otras áreas claves, y así, al oeste de la Cuenca de México, Sugiura (2005) y sus colegas realizaron un recorrido de $1500 \mathrm{~km}^{2}$ del valle de Toluca, en el cual registraron fuertes cambios en asentamiento asociado con la transición al Clásico, igual que en la Cuenca de México y en Puebla-Tlaxcala. En 1973, Hirth realizó un recorrido del valle del río Amatzinac, sobre un área aproximada de $450 \mathrm{~km}^{2}$, proporcionando una perspectiva regional sobre uno de los centros más importantes del Formativo Medio, Chalcatzingo, que, aunque se conectó a las redes de intercambio interregionales y contó con arte de estilo olmeca, no estuvo densamente poblado (Hirth 1987). Posteriormente, Hirth y colegas realizaron un recorrido de $560 \mathrm{~km}^{2}$ en la región de Tepeaca, ubicada en el sur de Puebla, utilizando métodos similares al recorrido de Amatzinac y permitiendo una comparación interregional controlada (Castanzo y Hirth 2008). Encontraron que, aunque la región de Tepeaca no contó con un solo centro tan grande como Chalcatzingo durante el periodo Formativo Medio, la población en la región aumentó fuertemente durante el Formativo Terminal, período durante el cual es posible hablar de varias sub-regiones del centro de México como urbanizadas.

En las Figuras 3 y 4 se pueden apreciar mapas con representaciones visuales mediante un montaje de los datos derivados de algunos de estos proyectos regionales. A ellos se añaden los asentamientos documentados recientemente por García Cook (2003, 2009) en una parte del Valle Oriental que incluye a Cantoná. Aunque se ha tratado de simplificar y estandarizar los sistemas de clasificación de estos variados proyectos, todavía deben ser entendidos como conjuntos de datos heterogéneos adquiridos a través de diversas metodologías, y que poseen deficiencias en su cobertura. Sin embargo, los mapas proporcionan medios visuales para comprender la demografía de este paisaje urbanizado durante los dos impulsos de urbanismo que caracterizan a los periodos Formativo y Clásico en el Altiplano Central.

\section{El sedentarismo y el urbanismo inicial del Formativo Temprano y Medio}

La cronología actual para la sedentarización el urbanismo de Altiplano Central durante el periodo Formativo se basa en los proyectos mencionados anteriormente, excavaciones recientes, nuevas fechas y calibraciones de radiocarbono, así como la comparación de secuencias sub-regionales basadas principalmente en la cerámica. En el Cuadro 1 se presenta una tabla comparativa, aunque se debe considerar que sigue en progreso el estudio de la cronología y que existe una falta de consenso en ciertas regiones, así como la necesidad de, en muchos casos, subdividir las fases. El gráfico pone de relieve el hecho de que ciertas regiones poseen cronologías cerámicas más profundas que otras, en su mayor parte como reflejo de los procesos graduales del poblamiento del paisaje por parte de comunidades de agricultores y alfareros durante el período Formativo Temprano. La apariencia inicial de las aldeas agrícolas se corresponde, en gran medida, con la productividad de nichos ecológicos, con los primeros habiendo aparecido en las zonas con mayor precipitación anual y proximidad a las fuentes de agua permanentes, como la tierra templada de Morelos y el sur de Puebla, y las regiones con lagos y/o humedales en el sur de la Cuenca de México y en el sur del Valle de Puebla-Tlaxcala. Áreas más áridas y alejadas de fuentes de agua permanentes probablemente fueron colonizadas por grupos que se instalaron de las zonas más productivas, aunque el grado de expansión por la migración de 


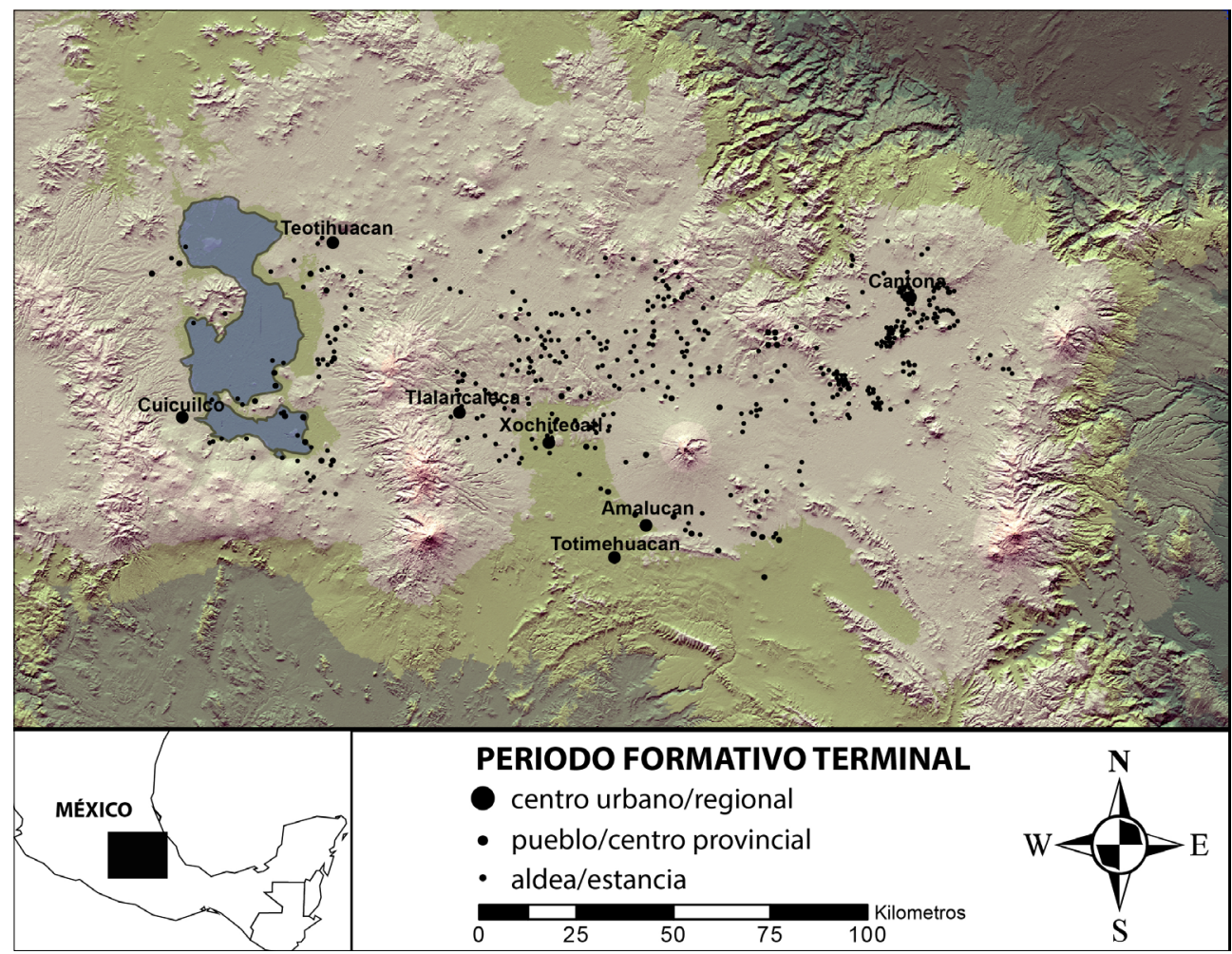

Figura 3. Asentamiento durante el Formativo Terminal basado en los recorridos de Sanders et al. (1979), García Cook $(1981,2009)$ y Castanzo y Hirth (2008).

poblaciones agricultores frente la adaptación autóctona de los grupos de cazadoresrecolectores sigue siendo tema de debate (Lesure 2008, 2014).

La arquitectura monumental más antigua en el centro de México se aprecia en sitios como Chalcatzingo, donde una plataforma del final del Formativo Temprano -que mide aproximadamente $15 \mathrm{~m}$ de largo- representa una primera etapa en una programa de elaboración llevado a cabo principalmente durante el Formativo Medio que alcanza los $70 \mathrm{~m}$ de longitud y $8 \mathrm{~m}$ de altura (Grove1987). En su recorrido de la región, Hirth (1987) estimó que Chalcatzingo llegó a cubrir 43,25 hectáreas ocupadas por entre 433 y 1.081 individuos, aproximadamente un tercio de la población estimada de los $450 \mathrm{~km}^{2}$ del estudio. Aunque no fue un centro urbano grande, Chalcatzingo fue sin duda una de las comunidades tempranas más dinámicas del centro de México, ya que sus habitantes participaban en las redes de intercambio a larga distancia y un programa escultórico y de relieve basado en el estilo interregional designado como olmeca. Vestigios de estas actividades también se han documentado en Zazacatla, Morelos, y en Teopantecuanitlan, Guerrero. Las comunidades de este cinturón de tierra templada de Guerrero, Morelos y la región de Las Bocas del sur de Puebla exhibieron la expresión más fuerte de los atributos iconográficos asociados con el estilo olmeca, también designado como Horizonte Temprano, del centro de México (Niederberger 1996). 


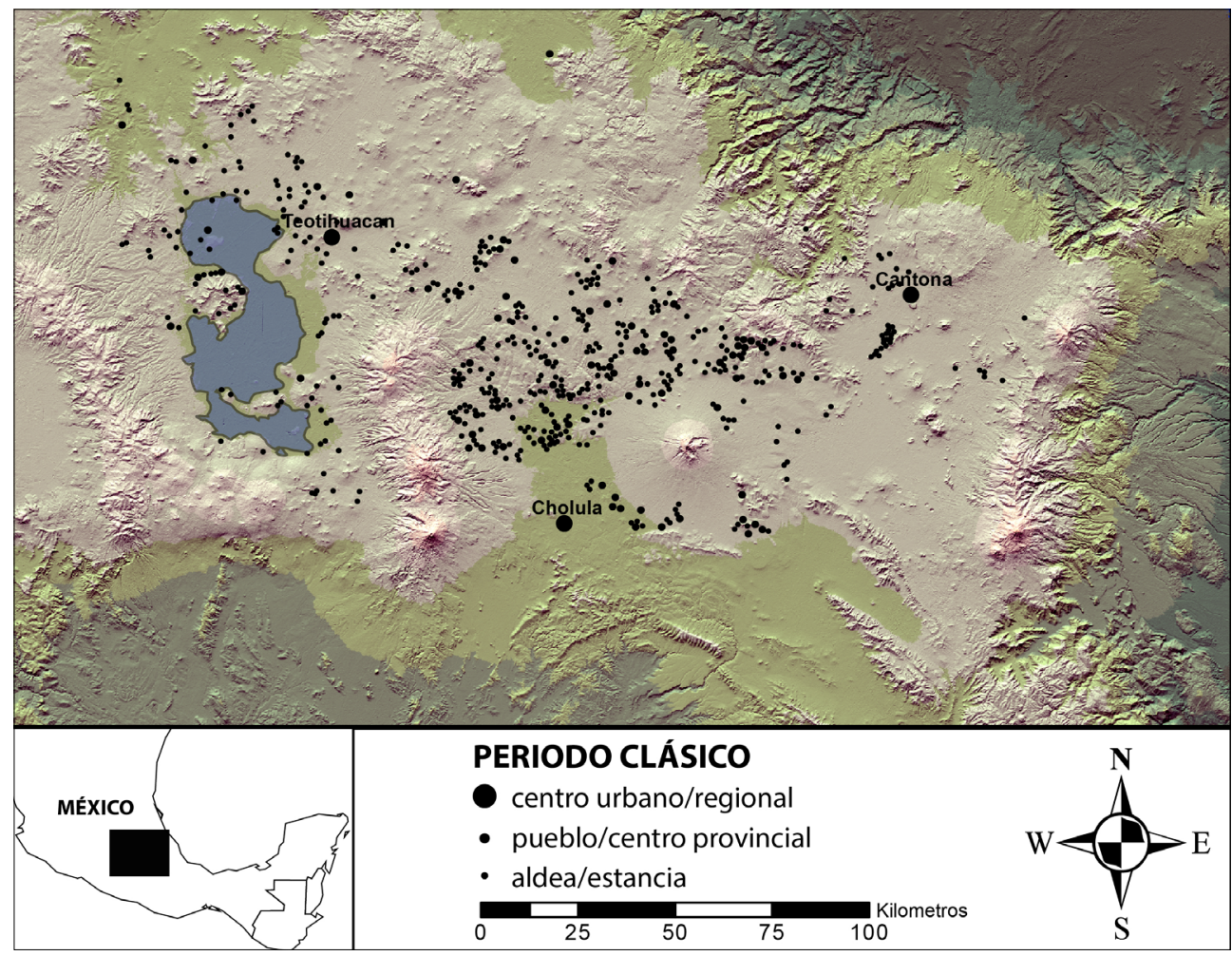

Figura 4. Asentamiento durante el Clásico basado en los recorridos de Sanders et al. (1979), García Cook (1981, 2009) y Castanzo y Hirth (2008).

Durante la primera mitad del período Formativo, las comunidades del centro de México participan en el intercambio a larga distancia de bienes y en las ideas sobre el mundo, que se materializan en cerámica, piedra verde y otros materiales. Sin duda existieron, en la mayoría de las comunidades, espacios para reuniones públicas, pero sólo fueron construidos arquitectónicamente en algunos casos, siendo claramente más pequeños que en los centros contemporáneos de la Costa del Golfo. A pesar de que en los asentamientos como Chalcatzingo se puede decir que han servido para funciones urbanas de una población más amplia, son pocos los que clasificarían a ese sitio o a cualquiera de sus contemporáneos como ciudades (Grove 2000). Las ciudades del centro de México en cambio fueron creadas durante un impulso inicial de urbanización a escala macro-regional durante la segunda mitad del Formativo, cuando varios epicentros monumentales dominados por conjuntos arquitectónicos ceremoniales se levantaron en un paisaje de múltiples ciudades y pueblos, con poblaciones regionales mucho más grandes que en fases anteriores. 
Cuadro 1. Cronología comparativa para el Formativo y el Clásico en el centro de México.

Referencias: Cowgill (2015), García Cook (1981, 2009), Grove (1987), Lesure et al. (2014),

Merino Carrión (1989), Niederberger (1987), Pastrana y Ramírez (2012),

Sanders et al. (1979), Serra Puche (2012), Tolstoy (1989).

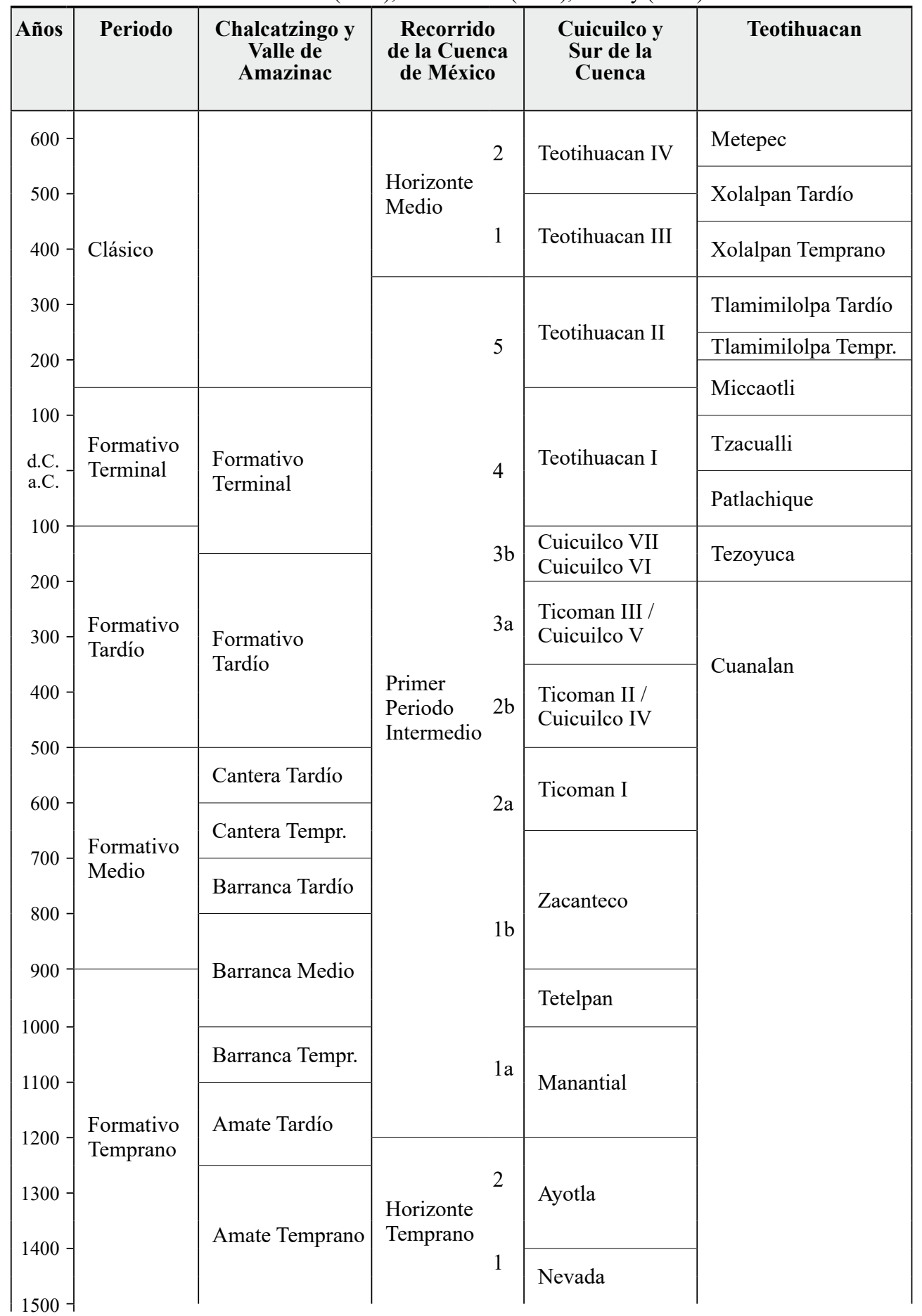


Cuadro 1 (continuación)

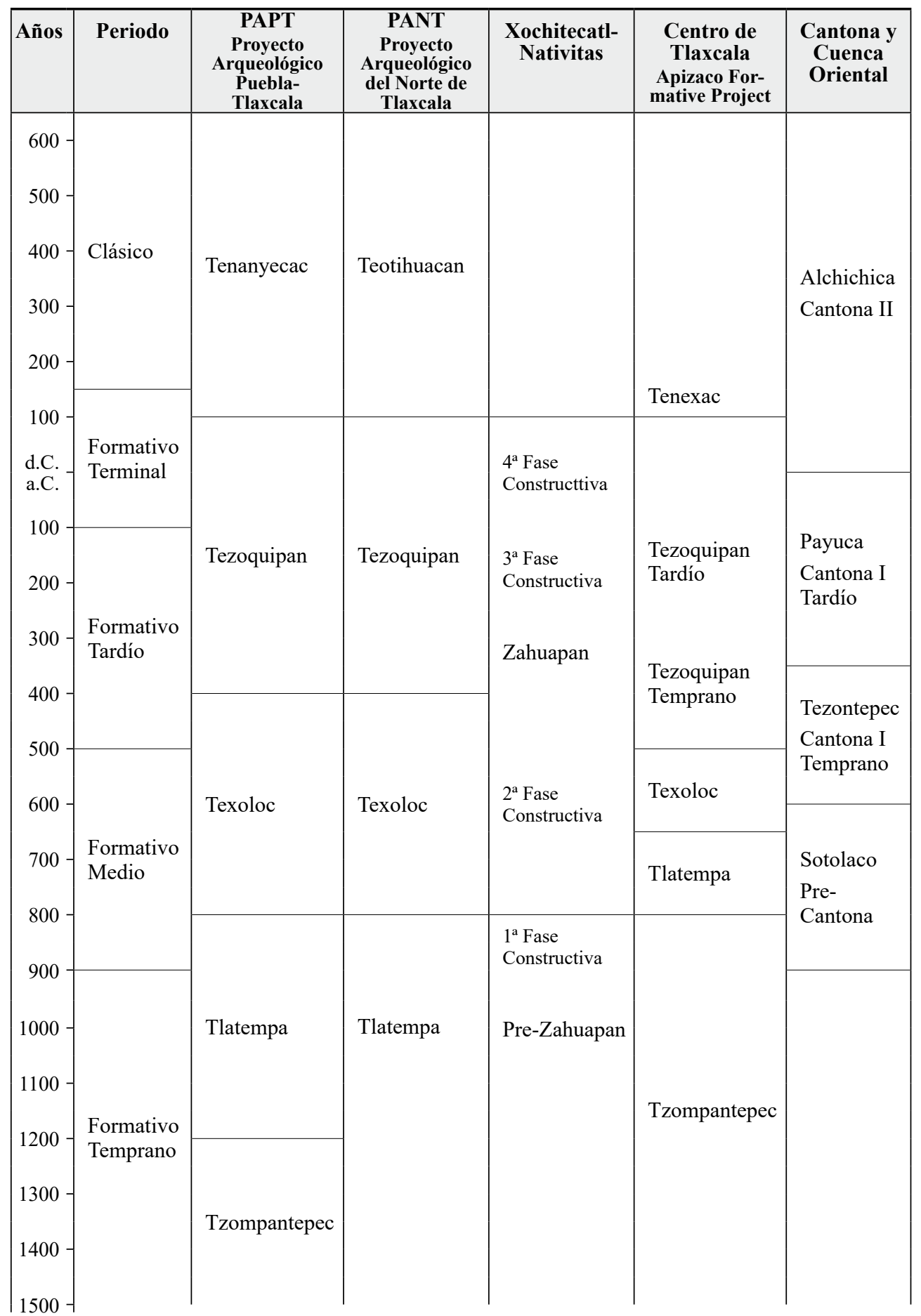




\section{Ciudades y dioses en el Formativo Tardío y Terminal}

La urbanización de la segunda mitad del Formativo se asoció con cambios en los patrones de intercambio y en las economías domésticas que Blanton et al. (2005) caracterizan como una «transformación de bienes regionales» centrado en la producción en masa de implementos utilitarios, en comparación con el enfoque en bienes de prestigio el cual caracterizó la primera mitad del Formativo. Cambios en los patrones regionales dentro de la Cuenca de México identifican al periodo entre 650-300 a.C., correspondiente a los finales del Formativo Medio y el inicio del Formativo Tardío, como el intervalo cuando se define por primera vez una jerarquía de asentamientos de tres niveles: 1) aldeas y pueblos pequeños (combinado para esta discusión), 2) pueblos grandes y 3) los centros regionales (ver Figura 3). Sanders et al. (1979: 97104) propusieron que el patrón o bien podría representar unas pocas jerarquías de asentamiento autónomas, o un sistema más grande con cuatro niveles de organización dominado por Cuicuilco, que se estima como un asentamiento de 5.000-10.000 personas. Estos autores sugieren que durante dos siglos del Formativo Tardío (ca. 300-100 a.C.) Cuicuilco creció hasta cubrir un mínimo de 400 hectáreas (Figura 5), con una población por encima de los 20.000 ocupantes.

Alejandro Pastrana y Felipe Ramírez (2012) proporcionan el resumen más actual y completo sobre Cuicuilco. Proponen una ocupación principal de $c a .800$ a.C.-250 d.C. con ocupaciones posteriores dispersas e intermitentes. Debido al hecho de que Cuicuilco está cubierta de lava, las causas de su abandono han sido relacionadas desde las primeras exploraciones con el vulcanismo. Investigaciones previas normalmente situaron el abandono de Cuicuilco durante los primeros siglos antes de nuestra era. El cono de ceniza Xitle, ubicado a unos $5 \mathrm{~km}$ al sudoeste, todavía parece haber producido la erupción devastadora que despobló el sitio, pero existe debate acerca de si esta erupción data del primer o del tercer siglo d.C. (Siebe 2000; UrrutiaFucugauchi et al. 2016). La temporalidad es importante porque en el primer caso la erupción podría haber sido causa directa de la urbanización de Teotihuacan, pero en el segundo caso Teotihuacan ya hubiera sido la ciudad más grande de las Américas.

Pastrana y Ramírez han descubierto lava almohadillada en el centro del sitio, entre Cuicuilco A y B, así como también en Cuicuilco D, ubicado al este. Tales formaciones se producen cuando la lava se enfría rápidamente por el agua, lo que indica que estas dos áreas habían tenido agua, ya sea en forma de embalses o lagunas. La presencia de manantiales y vestigios de canales en Cuicuilco $\mathrm{C}$ y otros canales al suroeste de Cuicuilco A, sugieren modificaciones hidráulicas significativas en estas áreas. Para quienes visitan el sitio en la actualidad, lo que se ve principalmente entre el pedregal y el marco urbano de la Ciudad de México son templos, incluyendo el basamento circular de Cuicuilco A. Sin embargo, se han excavado algunas unidades domésticas en Cuicuilco y la Estructura II podría representar un palacio o edificio administrativo.

Los períodos del Formativo Tardío y Terminal en el sur de Puebla-Tlaxcala eran iguales en su dinamismo a los desarrollos contemporáneos en la Cuenca de México. García Cook (1981) caracteriza a la mayoría de las áreas investigadas en sus recorridos durante el periodo como un florecimiento regional que terminó en declive demográfico y cultural durante el periodo Clásico, excepto en las zonas que rodean a las incipientes ciudades de Cholula y Cantoná, ambas a una distancia algo alejada del corazón del estado teotihuacano. Los asentamientos formativos también parecen 


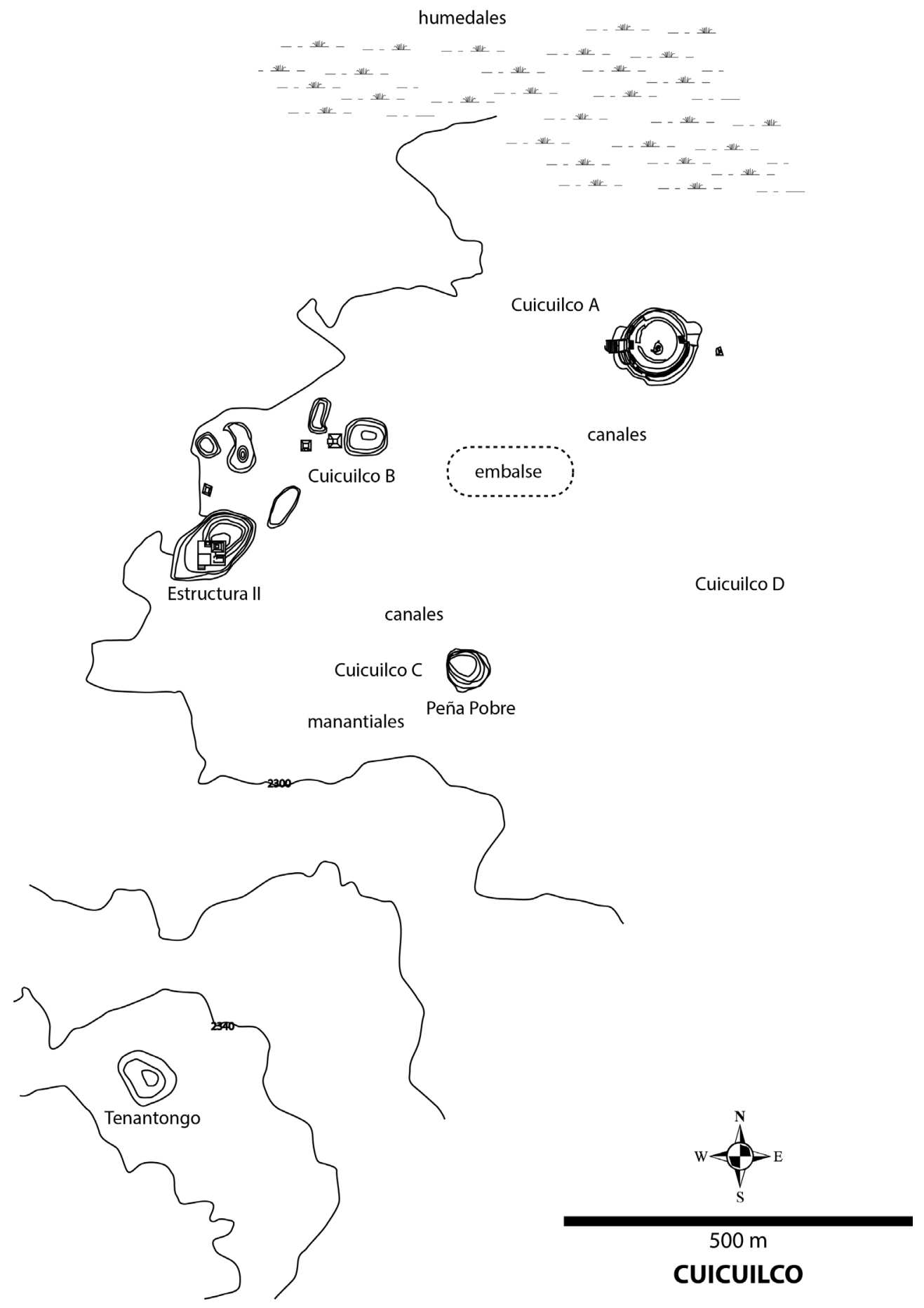

Figura 5. El sitio de Cuicuilco, basado en Mueller (1990), Pastrana y Ramírez (2012), Rodríguez Sánchez (1993) y Schávelzon (1983). 
haber sido densamente ocupados, pero es probable que el relativamente alto grado de nucleación documentado para la Cuenca de México no fuera igualado en PueblaTlaxcala, donde los asentamientos parecen haber sido mucho más dispersos. Sin embargo, hubo muchos centros de tamaño considerable en Puebla-Tlaxcala y en algunos la arquitectura monumental es equivalente o superior en su grandeza a la de Cuicuilco, como es el caso de Xochitecatl (Serra Puche 2012). Los epicentros urbanos demuestran variabilidad, con algunos enfocados en grandes pirámides centrales, mientras que otros elaboraron múltiples y dispersas agrupaciones templo-plaza, a veces combinándolas con una cancha para el juego de pelota.

Las altas y secas planicies del centro y norte de Tlaxcala y el sur de Hidalgo, como la región de Apan, no conocieron el desarrollo de un centro urbano formativo de la escala de los de fases contemporáneas de la Cuenca de México y el sur de PueblaTlaxcala. No obstante, sus habitantes estuvieron estrechamente relacionados con estas regiones y sus centros urbanos incipientes, ya que habitaban un corredor natural de comunicación entre el norte de la Cuenca de México y la costa del Golfo, y entre el valle de Puebla-Tlaxcala y fuentes de obsidiana del norte (Carballo y Pluckhahn 2007). Los sitios más grandes, como La Laguna, con sus funciones urbanas tuvieron capacidad para atraer a las poblaciones más rurales a su centro cívico-ceremonial para el ritual, el deporte, el comercio y otras actividades, pudiendo reunir a grandes grupos de personas. El sitio de La Laguna se encuentra al norte de la cima del volcán La Malinche, en el parteaguas continental, y abarca aproximadamente $1 \mathrm{~km}^{2}$ (Figura 6). Por ello lo consideramos un pueblo o centro urbano pequeño durante su apogeo (ca. 600 a.C.-150 d.C.) en comparación a los asentamientos más claramente identificados como ciudades o centros urbanos grandes del mismo nivel, o sea Cuicuilco, Xochitecatl, Teotihuacan, Cholula y Cantoná. Sin embargo, desde esta perspectiva de una zona menos urbanizada podemos apreciar los efectos macroregionales del urbanismo del Altiplano durante la transición entre el Formativo y el Clásico.

El hecho de que el sitio de La Laguna se ubique entre tres cerros con sus modestas estructuras cívico-ceremoniales posicionadas entre ellos, ha ocasionado la acumulación de depósitos coluviales y poca visibilidad de la arquitectura desde la superficie, con excepción de las estructuras más altas. Por ello, realizamos un programa de prospección geofísica para mapear los contornos arquitectónicos del recinto ceremonial y para seleccionar lugares idóneos dentro del recinto a fin de realizar excavaciones enfocadas al entendimiento de las actividades rituales (Carballo 2012, 2016; Carballo et al. 2014). La Plaza Central del sitio, consiste en una plaza rectangular con un basamento piramidal al lado este, un altar central y una cancha del juego de pelota al oeste. Este patrón se establece para los centros ceremoniales posteriores en el Altiplano en sitios como Cantoná, Tula y varios sitios aztecas.

Como otros grupos mesoamericanos, los habitantes de La Laguna hicieron ofrendas para ritualizar su ambiente construido, para consagrar y dar por concluido el uso vital de las estructuras. Asociado con la Estructura 12M-3, ubicada en la Plaza Este, recuperamos dos vasijas efigies casi completas con las características de dos seres sobrenaturales bien conocidos en fases posteriores y en algunos contextos contemporáneos a La Laguna (Figura 7). La primera (Figura 7a) representa al Dios Tormenta (una «vasija Tlaloc»), la cual fue dejada como ofrenda dentro de un cajón hecho de piedra y una mano de metate. Existen otros ejemplos de vasijas con el precursor formativo de este bien conocido dios que provienen de templos-basamentos, como en el sitio de Tlapacoya (Barba de Piña Chán 1980). La segunda (Figura 7b) 


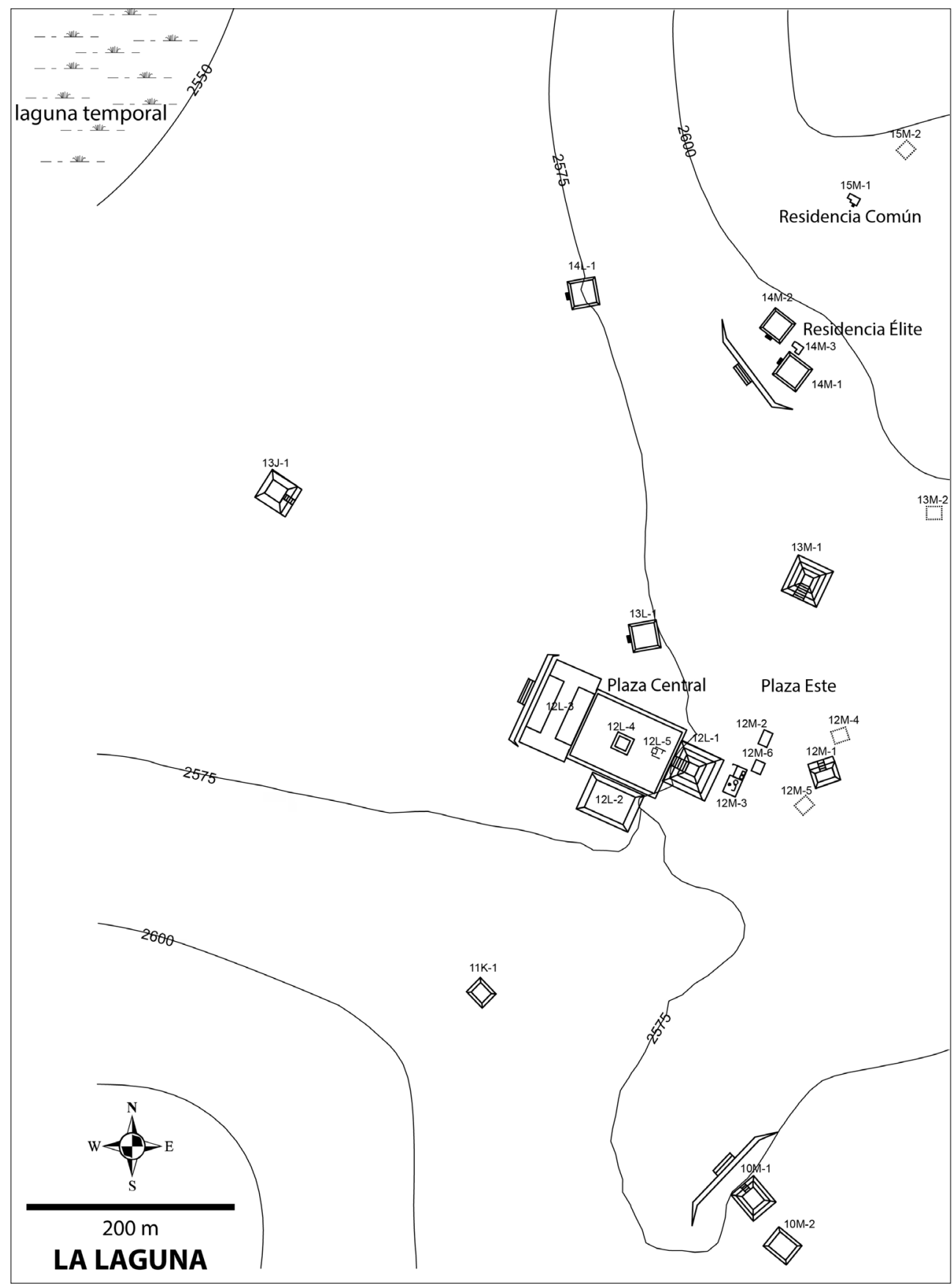

Figura 6. El sitio de La Laguna, basado en investigaciones del autor. 


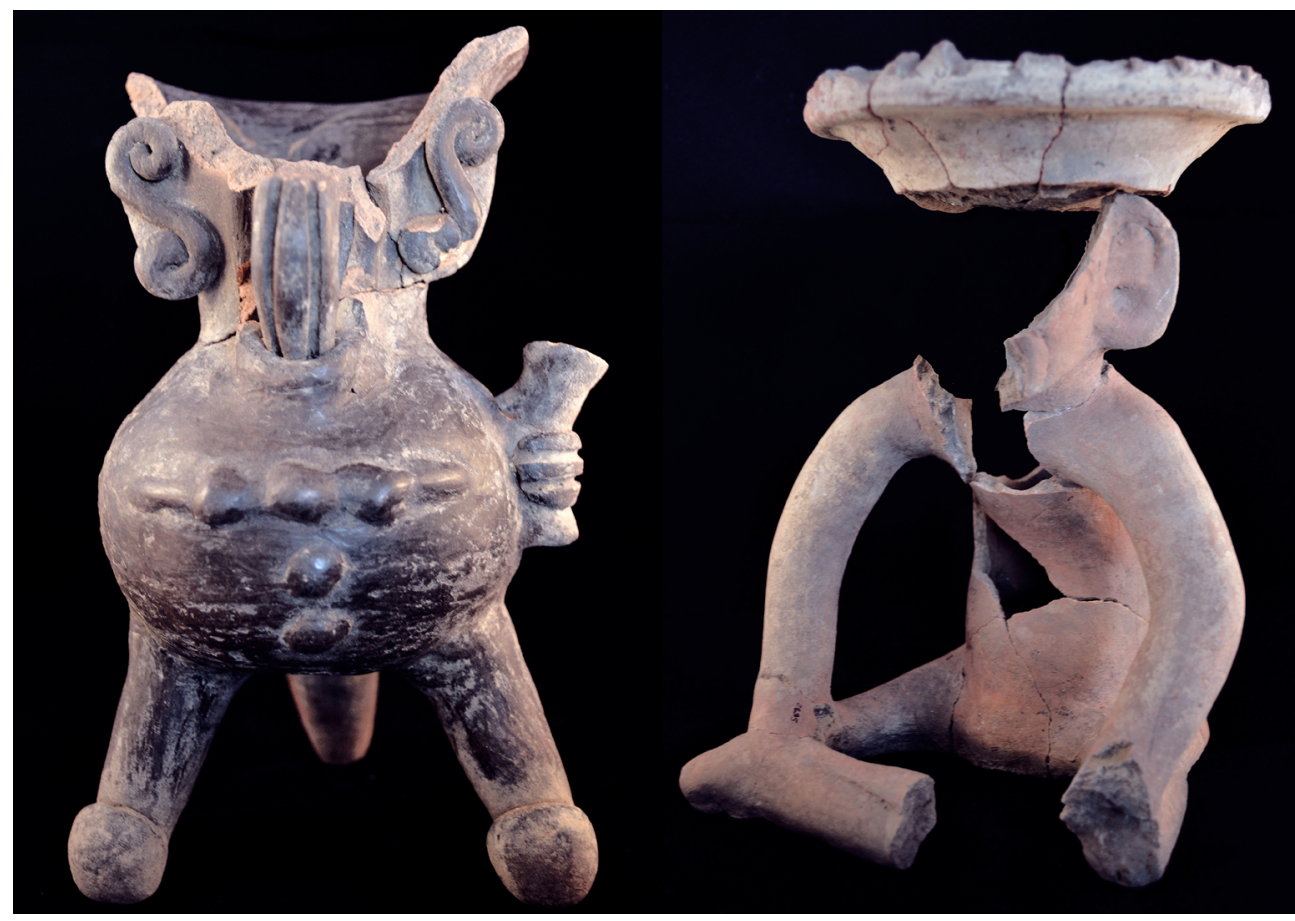

Figura 7. Vasijas efigie de la Estructura 12M-3 de La Laguna representando al Dios Tormenta (izquierda) y al Dios Viejo del Fuego (derecha).

representa al Dios Viejo del Fuego (un «incensario Huehueteotl»), la cual fue dejada sobre el piso dentro de un cajón o cuarto muy reducido en la parte posterior de la estructura. Se conocen representaciones formativas de este dios en Cuicuilco y otros sitios (Carballo 2007). Juntos, los dioses representan el agua y el fuego, un dualismo clave en la cosmovisión del Altiplano, expresado por el concepto atl-tlachinolli para los mexicas. El hecho de que ambas efigies fueran desfiguradas puede corresponder a una terminación ritual de la fuerza vital de estas efigies, ya que esos elementos claves de las piezas no se encontraron en la estructura y pueden haber sido depositados en otro lugar. Esta práctica ritual es conocida entre grupos indígenas actuales (McGee 1998).

Como complemento a estas representaciones de dioses, los habitantes de La Laguna fabricaron otros elementos y realizaron ofrendas incluyendo materiales como espejos o reflectores de pizarra con pigmento, excéntricos o siluetas de obsidiana y otros materiales que llegaron desde largas distancias, como concha marina y jade (Carballo 2016). Estas actividades fueron también realizadas en otros sitios de la región y sentaron las bases de la codificación de ciertas conceptualizaciones y materializaciones de la religión elaboradas por las civilizaciones posteriores. Sin embargo, en la transición entre los patrones urbanos formativos y clásicos hubo también transformaciones clave en los modos de la vida en el Altiplano. 


\section{Las transiciones al período Clásico}

La transición del paisaje urbano entre el Formativo y el Clásico registró cambios dramáticos en los patrones de asentamiento y demográficos. Algunas partes de PueblaTlaxcala y el sur de la Cuenca de México tuvieron significativas disminuciones de población en correspondencia con incrementos demográficos en Cholula y Teotihuacan que sugieren procesos de migración a estas ciudades. Un ejemplo se representa en la Figura 8, que recopila datos derivados de los recorridos arqueológicos llevados a cabo en el norte de Tlaxcala y el Valle de Teotihuacan y publicados, respectivamente, por Merino Carrión (1989) y por Gorenflo y Sanders (2007). Vistos juntos, los patrones de asentamiento en las dos sub-regiones son bastante similares durante el período Formativo Tardío. Sin embargo, mientras que La Laguna fue un pueblo significativamente mayor en tamaño (calculado en hectáreas) que sus contemporáneos, hubo tres asentamientos en el Valle de Teotihuacan clasificados como grandes aldeas nucleadas que no se distinguen tan claramente si se considera el tamaño del área y la población estimada. Si la población se considera más que el tamaño del
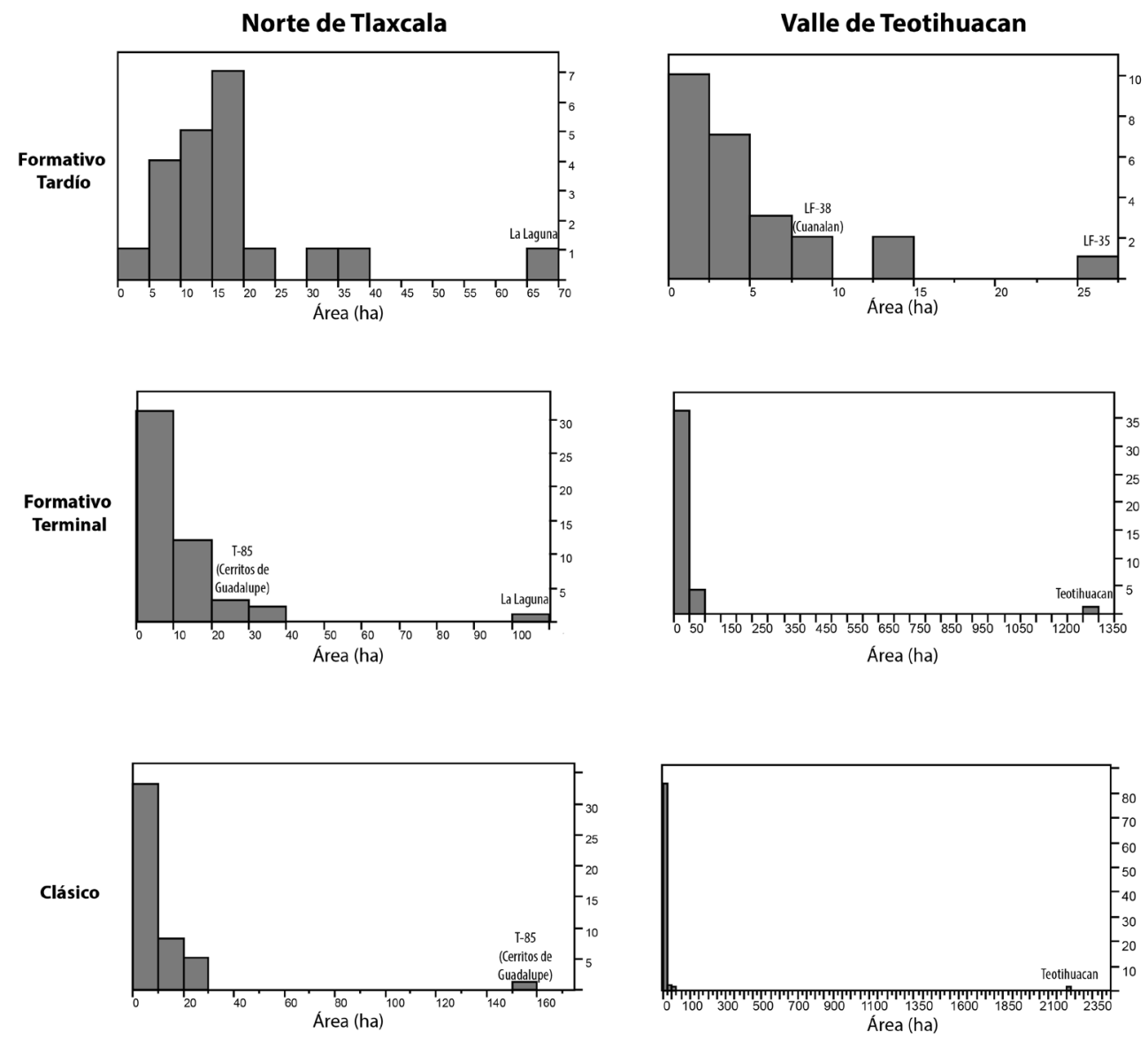

Figura 8. Histogramas de asentamientos en el norte de Tlaxcala (modificado de Merino Carrión [1989]) y el Valle de Teotihuacan (modificado de Gorenflo y Sanders [2007]). 
lugar, Cuanalán podría ser mayor que LF/TF-35, un sitio ubicado cerca de los manantiales en el Valle de Teotihuacan. Sin embargo, Cowgill (2015: 48-49) propone que LF/TF-35 también tuvo una población mayor que Cuanalán, la cual estima que alcanzó entre 1000-2000 habitantes. En cualquiera caso, estos sitios mayores de la época en el Valle de Teotihuacan fueron de un tamaño similar, menor que La Laguna, y probablemente representan asentamientos independientes.

La urbanización teotihuacana creó una jerarquía híper-primada o principal ( $h y$ per-primate $)^{2}$ de asentamientos en el valle durante el Formativo Terminal y el Clásico, y sus efectos se pueden reconocer también en el norte de Tlaxcala. Dentro de su sub-región, la jerarquía del Norte de Tlaxcala exhibe un patrón más consistente con los centros urbanos y sus zonas de influencia reducidas, pero visto desde la perspectiva de la macro-región del centro de México, se aprecia cómo se entrelaza la evolución de las dos sub-regiones. Después de continuar en la cima de la jerarquía de asentamientos durante el Formativo Terminal, La Laguna fue abandonada durante la transición clásica (Carballo 2009, 2012), y un asentamiento designado como T-85 (Cerritos de Guadalupe, previamente de segundo rango), se convirtió en el centro de mayor tamaño. Este sitio se encuentra directamente en el corredor natural de comunicación entre Teotihuacan y la Costa del Golfo (Carballo y Pluckhahn 2007) y muestra afinidades materiales con Teotihuacan (Merino 1989), lo que indica que el crecimiento de la ciudad tuvo un efecto dominó en una zona de influencia externa. La comparación entre los patrones de asentamiento subraya como el urbanismo pre-moderno, visto en el registro arqueológico, no se puede entender sólo desde la perspectiva de las ciudades $u$ otros centros urbanos. Por el contrario, requiere perspectivas macro-regionales las cuales incluyen áreas periféricas al desarrollo de las ciudades y los estados mayores. Tales zonas periféricas podrían haber sido esenciales para el comercio y así contribuir a los procesos por los cuales se construyeron las identidades culturales macro-regionales. Aunque las transformaciones entre el Formativo y Clásico fueron fuertes, la continuidad entre los dos periodos tomó muchas formas. Uruñuela y Plunket (2007; Plunket y Uruñuela 2012) trazan los orígenes formativos de elementos esenciales en la vida urbana en Teotihuacan incluyendo las fachadas arquitectónicas de forma talud-tablero, complejos de tres estructuras rodeando un patio con altares centrales y la cerámica fina e importada llamada Anaranjada Delgada. Visto desde una perspectiva macro-regional, que toma en cuenta un centro como el Valle de Teotihuacan y una periferia como el norte de Tlaxcala, se pueden apreciar los procesos co-evolucionarios en la arquitectura ceremonial, los símbolos religiosos y las prácticas rituales, los cuales definieron los patrones urbanos y religiosos vistos en ciudades del período Clásico y en las sociedades posteriores.

\section{Referencias}

Barba de Piña Chán, Beatriz

1980 Tlapacoya: los principios de la teocracia en la Cuenca de México. México: Biblioteca Enciclopédica del Estado de México.

\footnotetext{
2 Término procedente de la geografía urbana. Fue desarrollado por el geógrafo Mark Jefferson en los años 30s para explicar el hecho de que grandes ciudades aglutinen un gran porcentaje de población de un país o región, así como su actividad económica.
} 
Blanton, Richard E., Lane F. FARGHer y Verenice Y. Heredia EsPinOza

2005 «The Mesoamerican World of Goods and its Transformations», en Settlement, Subsistence, and Social Complexity: Essays Honoring the Legacy of Jeffrey R. Parsons, Richard E. Blanton, ed., pp. 260-294. Los Angeles: Cotsen Institute of Archaeology, University of California.

Carballo, David M.

2007 «Effigy Vessels, Religious Integration, and the Origins of the Central Mexican Pantheon». Ancient Mesoamerica 18 (1): 53-67.

2009 «Household and Status in Formative Central Mexico: Domestic Structures, Assemblages, and Practices at La Laguna, Tlaxcala». Latin American Antiquity 20 (3): 473-501.

2012 «Public Ritual and Urbanization in Central Mexico: Plaza and Temple Offerings from La Laguna, Tlaxcala». Cambridge Archaeological Journal 22 (3): 329-352.

2016 Urbanization and Religion in Ancient Central Mexico. Nueva York: Oxford University Press.

Carballo, David M. y Thomas Pluckhahn

2007 «Transportation Corridors and Political Evolution in Highland Mesoamerica: Settlement Analyses Incorporating GIS for Northern Tlaxcala, Mexico». Journal of Anthropological Archaeology 26 (4): 607-629.

Carballo, David M., Luis Barba, Agustín Ortíz, Jorge Blancas, Nicole Cingolani, Jorge

Toledo Barrera, David Walton, Isabel Rodríguez López y Lourdes CouoH

2014 «Suprahousehold Consumption and Community Ritual at La Laguna, Mexico». Antiquity 88 (339): 141-159.

Castanzo, Ronald A. y Kenneth G. HiRth

2008 «El asentamiento del periodo Formativo en la cuenca central de Puebla-Tlaxcala, México», en Ideología política y sociedad en el periodo Formativo: ensayos en homenaje al doctor David C. Grove, Ann Cyphers y Kenneth G. Hirth, eds., pp. 203-231. México: Instituto de Investigaciones Antropológicas, Universidad Nacional Autónoma de México.

CowgILl, George L.

2015 Ancient Teotihuacan: Early Urbanism in Central Mexico. Nueva York: Cambridge University Press.

García CoOK, Ángel

1973 «Algunos descubrimientos en Tlalancaleca, Estado de Puebla». Comunicaciones 9: 25-34. Puebla: Fundación Alemana de Investigación Científica.

1976 «El Proyecto Arqueológico Puebla-Tlaxcala: origen, finalidad y logros». Suplemento de Comunicaciones 3: 5-12. Puebla: Fundación Alemana de Investigación Científica.

1981 «The Historical Importance of Tlaxcala in the Cultural Development of the Central Highlands», en Supplement to the Handbook of Middle American Indians, Vol. 1, Archaeology, Jeremy A. Sabloff, ed., pp. 244-276. Austin: University of Texas Press.

2003 «Cantona: The City», en Urbanism in Mesoamerica, Volume 1, William T. Sanders, Alba Guadalupe Mastache y Robert H. Cobean, eds., pp. 311-343. México y University Park: Instituto Nacional de Antropología e Historia y Pennsylvania State University. 
2009 «El Formativo en la mitad norte de la Cuenca de Oriental». Arqueología 40: 115152.

2014 «Importancia de la región poblano-tlaxcalteca en el surgimiento de las grandes ciudades del Altiplano central de México». Arqueología 49: 91-104.

Gorenflo, Larry J. y William T. SANDERS

2007 Archaeological Settlement Pattern Data from the Cuautitlan, Temascalapa, and Teotihuacan Regions, Mexico. University Park: Department of Anthropology, Pennsylvania State University.

Grove, David C.

2000 «The Preclassic Societies of the Central Highlands of Mesoamerica», en The Cambridge History of the Native Peoples of the Americas, Vol. II, Mesoamerica, Part 1, Richard E. W. Adams y Murdo J. Macleod, eds., pp. 122-155. Nueva York: Cambridge University Press.

Grove, David C. (ed.)

1987 Ancient Chalcatzingo. Austin: University of Texas Press.

HiRTH, Kenneth G.

1987 «Formative Period Settlement Patterns in the Río Amatzinac Valley», en Ancient Chalcatzingo, David C. Grove, ed., pp. 343-367. Austin: University of Texas.

LESURE, Richard G.

2008 «The Neolithic Demographic Transition in Mesoamerica? Larger Implications of the Strategy of Relative Chronology», en The Neolithic Demographic Transition and its Consequences, Jean-Pierre Bocquet-Appel y Ofer Bar-Yosef, eds., pp. 107138. Nueva York: Springer.

2014 «Macroregional Research Topics for Formative Central Tlaxcala», en Formative Lifeways in Central Tlaxcala, Volume 1: Excavations, Ceramics, and Chronology, Richard G. Lesure, ed., pp. 363-370. Los Angeles: Cotsen Institute of Archaeology Press, University of California.

Lesure, Richard G., Isabel Rodríguez López, Aleksander Borejsza, Jennifer Carballo y David M. CARBALlo

2014 «Pottery: Description and Typology», en Formative Lifeways in Central Tlaxcala: Volume 1, Excavations, Contexts, and Chronology, Richard G. Lesure, ed., pp. 183258. Los Angeles: Cotsen Institute of Archaeology Press, University of California.

MCGEE, R. Jon

1998 «The Lacandon Incense Burner Renewal Ceremony: Termination and Dedication Ritual among the Contemporary Maya», en The Sowing and the Dawning: Termination, Dedication, and Transformation in the Archaeological and Ethnographic Record of Mesoamerica, Shirley Boteler Mock, ed., pp. 41-46. Albuquerque: University of New Mexico Press.

MERINO CARrión, Beatriz Leonor

1989 La cultura Tlaxco. México: Instituto Nacional de Antropología e Historia.

Niederberger Betton, Christine

1987 Paleopaysages et archeologie pre-urbaine du Bassin de Mexico, Vol. I-II. México: Centro de Estudios Mexicanos y Centroamericanos.

1996 «Olmec Horizon Guerrero», en Olmec Art of Ancient Mexico, Elizabeth P. Benson y Beatriz de la Fuente, eds., pp. 95-103. Washington DC: National Gallery of Art. 
Pastrana, Alejandro y Felipe Ramírez

2012 «Reinterpretando Cuicuilco». Ponencia presentada en el 77rd Annual Meeting de la Society of American Archaeology, Memphis, Tennessee.

PlunKeT, Patricia y Gabriela URUÑUela

2005 «Recent Research in Puebla Prehistory». Journal of Archaeological Research 13 (2): 89-127.

2012 «Where East Meets West: The Formative in Mexico's Central Highlands». Journal of Archaeological Research 20 (1): 1-51.

RoDRÍGUEZ SÁNCHEZ, Ernesto

1993 «Cuicuilco «C»: aportes sobre aspectos urbano-arquitectónicos en el Formativo de la Cuenca de México», en A propósito del Formativo, Ma. Teresa Castillo Mangas, ed., pp. 59-72. México: Instituto Nacional de Antropología e Historia.

SÁnchez-PÉrez, Serafín, Elizabeth Solleiro-Rebolledo, Sergey Sedov, Emily McClung De Tapia, Alexandra Golyeva, Blanca Prado y Emilio Ibarra-Morales

2013 «The black San Pablo paleosol of the Teotihuacan Valley, Mexico: Pedogenesis, fertility, and use in ancient agricultural and urban systems». Geoarchaeology 28 (3), 249-267.

SANDERs, William T., Jeffrey R. PARsons y Robert S. SANTLEY

1979 The Basin of Mexico: Ecological Processes in the Evolution of a Civilization. Nueva York: Academic Press.

SCHÁVELZON, Daniel

1983 La pirámide de Cuicuilco: álbum fotográfico, 1922-1980. México: Fondo de Cultura Económica.

SERra PuCHe, Mari Carmen

2012 Xochitécatl. Segunda edición. Tlaxcala: Instituto Tlaxcalteca de la Cultura.

SIEBE, Claus

2000 «Age and Archaeological Implications of Xitle Volcano, Southwestern Basin of Mexico-City». Journal of Volcanology and Geothermal Research 104: 45-64.

Sugiura, Yoko

2005 Y atrás quedó la ciudad de los dioses: Historia sobre los asentamientos en el valle de Toluca. México: Instituto de Investigaciones Antropológicas, Universidad Nacional Autónoma de México.

Tolstoy, Paul

1989 «Coapexco and Tlatilco: Sites with Olmec Materials in the Basin of Mexico», en Regional Perspectives on the Olmec, Robert J. Sharer y David C. Grove, eds., pp. 85-121. Nueva York: Cambridge University Press.

Tolstoy, Paul, Suzanne K. Fish, Martin W. Boksenbaum, Kathryn Blair Vaughn y C. Earle SMITH

1977 «Early Sedentary Communities of the Basin of Mexico». Journal of Field Archaeo$\log y$ (1): 91-106.

Urrutia-Fucugauchi, Jaime, Avto Goguitchaichvili, Ligia Pérez-Cruz y Juan MoraLES

2016 «Archaeomagnetic Dating of the Eruption of Xitle Volcano, Basin of Mexico: Implications for the Mesoamerican Centers of Cuicuilco and Teotihuacan». Arqueología Iberoamericana 30: 23-29. 
Uruñuela, Gabriela y Patricia Plunket

2007 «Tradition and Transformation: Village Ritual at Tetimpa as a Template for Early Teotihuacan», en Commoner Ritual and Ideology in Ancient Mesoamerica, Nancy Gonlin y Jon C. Lohse, eds., pp. 33-54. Boulder: University Press of Colorado. 